\title{
Effects of breastfeeding on body composition and maturational tempo in the rat
}

Yonatan Crispel ${ }^{1,3}$, Oren Katz ${ }^{1}$ Dafna Ben-Yosef ${ }^{2}$ and Ze'ev Hochberg ${ }^{1,3^{*}}$

\begin{abstract}
Background: Features of life history are subject to environmental regulation in the service of reproductive fitness goals. We have previously shown that the infant-to-childhood transition reflects the adaptive adjustment of an individual's size to the prevailing and anticipated environment.

Methods: To evaluate effects of weaning age on life-history traits in rats, we repeatedly measured length and body mass index (BMI), as well as physiological development and sexual maturation in pups weaned early (d16), normally (d21) or late (d26). Males were bred to females of the same weaning age group for four generations.

Results: Here, we show that the age at weaning from lactation regulates a rat's life history, growth, body composition and maturational tempo. We show that early-weaned rats developed faster than normal- or lateweaned rats; they are leaner and longer than late-weaned ones who are heavier and shorter. Early-weaned progeny develop more rapidly (that is, fur budding, pinnae detachment, eye opening); females show earlier vaginal opening and estrous and males show earlier onset of testicular growth. In generations 3 and 4, early-weaned rats bear larger litter sizes and heavier newborn pups. The entire traits complex is transmitted to subsequent generations from the paternal side.
\end{abstract}

Conclusions: The findings presented here lend support to the proposition that the duration of infancy, as indexed by weaning age, predicts and perhaps programs growth, body composition, and the tempo of physiological development and maturation, as well as litter size and parity and, thereby, reproductive strategy.

Keywords: growth, puberty, maturation, lactation, body composition, rat

\section{Background}

A multiplicity of factors and forces, including genetic, environmental and stochastic ones, influence how organisms grow and develop. This is so with respect to reproductively significant traits, a primary focus of this report, such as maturational tempo and pubertal timing [1]. Lifehistory theory is a powerful evolutionary framework $[2,3]$ for understanding a second focus of this essay: adaptive development plasticity, particularly with regard to growthand metabolic-related strategies for transitioning from one life-history phase to the next [4-6].

When it comes to the environmental regulation of life history, the time of maximal developmental plasticity appears to be during the prenatal and early postnatal

\footnotetext{
* Correspondence: z_hochberg@rambam.health.gov.l

'Division of Pediatric Endocrinology, Meyer Children's Hospital, Rambam

Health Care Campus, Haaliya Street, Haifa 31096, Israel
}

periods [6]. Indeed, the infancy-to-childhood transition (ICT) is often a time of heightened nutritional stress and mortality in humans, thereby representing a bottleneck for evolutionary forces. In social mammals, including apes, as well as in traditional human societies, the ICT is the time of weaning from breastfeeding, a third important focus here, demarcating the transition from maternal provision, protection and support to greater independence. Weaning from lactation is itself responsive to sex, stress and other environmental cues [7] that are presumed to inform the developing organism about risks and opportunities in its current and future environment. As such, the timing of the ICT has been hypothesized to reflect the adaptive adjustment of a species or an individual's size to the prevailing and anticipated environment, as the ICT is a major determinant of final adult height [5].

This view, that weaning is regulated by contextual conditions and serves to regulate life history in the Full list of author information is available at the end of the article

C Biomed Central 
service of fitness goals, led us to test experimentally in rats the proposition that the age of weaning, our index of ICT, provides cues for growth, maturation, developmental tempo and litter size, thereby affecting these outcomes. We also evaluate the proposition that such effects of weaning age are enhanced across generations in rats.

To evaluate effects of weaning age on life-history traits in rats, as well as on their offspring's development, we repeatedly measured length and BMI, as well as physiological development and sexual maturation in pups weaned early (d16), normally (d21) or late (d26). Due to concerns that the effects of maternal care on behavior could be reversed by cross-fostering [8,9], steps were taken to eliminate this possibility: weaning was accomplished through cross-fostering by non-lactating mothers, and separation was carried out on $\mathrm{d} 30$ for all. By the nature of that design, timing of weaning is confounded by time spent with a foster mother. Upon removal of lactating mothers, food was supplied $a d l i b$ as both chow and powder to ascertain its reach by all pups.

\section{Methods}

\section{Animals}

Gestating outbred Sprague-Dawley mother rats from timed pregnant colonies were housed at the Animal Facility of the Technion Faculty of Medicine (F0 generation). Delivered F1 generation offspring pups were diluted on Day 3 (d3) to include four female and four male pups for each mother. F1 males were bred to F1 females of the same weaning age group but from different litters, generating the F2 generation; F2 males were bred to F2 females to generate the F3 generation and the F3 were bred in the same manner to generate the F4 generations. No inbreeding or sibling crosses were generated. All animals were grown uninterrupted other than for weekly measurements until weaning on the designated $\mathrm{d} 16, \mathrm{~d} 21$ or $\mathrm{d} 26$. The entire experiment from F0 to F2 was performed twice. On weaning day, mothers were removed and mothers that had weaned litters successfully in the recent month were introduced as foster mothers [10]. Upon weaning, both chow cubes and chow powder were introduced to cages to ascertain $a d l i b$ feeding by young pups. The number of female pregnancies used for replicates in each weaning group on each generation was three, to provide 12 males and 12 females per group

\section{Developmental milestones}

Developmental milestones for infantile (pre-weaned) rats were observed daily from $\mathrm{d} 7$ to 20 [11]: incisor eruption (d7 milestone), fur budding (d11 milestone), eye opening (d13) and pinnae detachment (d15). Rat BMI was calculated as body weight over cubic rump-tail length $(\mathrm{gr} / \mathrm{cm} 2)$.

\section{Pubertal maturation}

Vaginal opening was determined by supine observation as of $\mathrm{d} 30$, showing closed vaginal cavity before and opened vaginal cavity after. First estrus was determined as of d36 by observing a vaginal smear daily for 15 days between 9 and 10 AM. Vaginal smears were prepared by introducing a drop of distilled water into the vagina, collecting back and placing it on a clean slide after adding a drop of glycerin. Estrus phase was confirmed when the smear showed more than $50 \%$ cornified epithelial cells. Testicular size was determined daily as of d30 using a self-built orchidometer based on the human Prader orchidometer [12] with mock-ups ranging from 0.5 to $5 \mathrm{ml}$. Testes measuring more than $1 \mathrm{ml}$ were considered pubertal.

\section{Body composition}

For assessment of body composition in newborn rats, we used the Minispec live mice TD-NMR Analyzer (Bruker LF50, Ettlingen, Germany) - a mice magnetic resonance apparatus for animals up to $15 \mathrm{~g}$ [13]. The onboard electronics calculate whole-body water, fat and lean mass. Because of the design characteristics of the instrument, lean mass is most highly correlated with skeletal muscle. Unanaesthetized animals were placed into a cylindrical holder and inserted into a receiving port on the machine for less than two minutes, allowing scans in triplicate.

\section{Enteral glucose tolerance test [14]}

The test was performed after six hours fasting around $2 \mathrm{pm} .0 .1 \mathrm{~g} / \mathrm{ml}$ glucose was given intragastric through a feeding tube to provide $1 \mathrm{~g}$ glucose for each $100 \mathrm{~g}$ body weight. A drop of blood was taken from the cut tail vein before the glucose load and after 15, 30, 45 and 60 minutes for the determination of blood glucose with a glucometer.

\section{Insulin Tolerance Test [14]}

The test was performed on random-fed rats around $2 \mathrm{pm}$. The rats were injected with insulin $(0.75 \mathrm{U} / \mathrm{kg})$ in $0.1 \mathrm{ml} 0.9 \% \mathrm{NaCl}$ intraperitoneally. A drop of blood was taken from the cut tail vein before the injection of insulin and after 15, 30, 45 and 60 minutes for the determination of blood glucose with a glucometer.

\section{Rat GH and IGF1}

Serum levels of rat GH and rat IGF1 were measured using the MG100 Rat/Mouse Growth Hormone and Rat IGF-I Quantikine ELISA Kit (R\&D Systems, Biotest, Solihull, West Midlands, UK).

\section{Statistical analysis}

Data were analyzed using an SAS program (SAS Institute Inc., Cary, NC. USA). The values were expressed as means and standard deviation (SD) or standard error of 
mean (SEM), as indicated. Statistical analysis was performed and the difference among the means of three groups was determined using two-way Analysis of Variance (ANOVA). A statistically significant difference was confirmed at $P<0.05$. We did not control for litter effects.

\section{Ethical approvals}

All animal procedures have been approved by the Technion Animal Use and Care Committee and were performed under the supervision of an experimental animal veterinarian surgeon.

\section{Results}

\section{Growth of early- or late-weaned animals}

Results revealed that weaning age influences the length and adiposity of rats from infancy through adulthood, with short lactation resulting in a thin/long phenotype and long lactation in a short/heavy phenotype. Specifically, earlyweaned (d16) F1 male and female rats were longer and late-weaned (d26) shorter relative to d21-weaned control animals. This was true from d30 to 90 , with the difference emerging between d19 and 30 (Figure 1a, b). Long d16 rats were thinner by BMI and short $\mathrm{d} 26$ animals were heavier than d21-weaned control animals (Figure 1c, d). Food consumption varied between the groups, as did endocrine measurements. Average ( \pm SD) adult daily food intake was $10.0 \pm 0.3 \mathrm{~g}$ for d16 rats, $10.6 \pm 0.2 \mathrm{~g}$ for $\mathrm{d} 21$ and $11.2 \pm$ $0.3 \mathrm{~g}$ for the short/heavy d26 male rats $(P<0.01$ vs. d16). Although serum growth hormone $(\mathrm{GH})$ of the two groups did not differ, serum IGF-1 did, being greater in lateweaned (short/heavy) rats $(0.57 \pm 0.20)$ as compared to early-weaned ones $(0.38 \pm 0.17 \mathrm{ng} / \mathrm{ml}, P=0.028)$.

In all growth parameters, the $\mathrm{d} 16$ group differed more than the $\mathrm{d} 26$ group from the $\mathrm{d} 21$ controls; therefore, we highlight especially the unique phenotype of early-weaned animals. The long/thin habitus of the d16-weaned group was associated in adulthood (d80 to 90) with greater glucose tolerance and insulin sensitivity, as compared to d26 rats, as determined by an enteral glucose tolerance test and an insulin tolerance test; Figure $2 \mathrm{a}$, b shows these differences in males, with similar results emerging in females

The long/thin phenotype of early-weaned F1 animals was sustained across generations but not intensified, with the same being true of the short/heavy phenotype of lateweaned F1 animals. This was determined after F1 and F2 male rats were mated on $\mathrm{d} 60$ to 70 with F1 to F2 females of the same weaning age group from different litters, with the weaning age of each group maintained for F2 to F3 pups.

\section{Infantile body composition}

To test the hypothesis that being a member of the lateweaned lineage (short/heavy/insulin resistant) is associated with infantile wasting, body composition of F3 pups was assessed on d10 by TD-NMR live mice Analyzers NMR (Minispec, Bruker LF50, Ettlingen, Germany), where lean mass is highly correlated with skeletal muscle [13]. Offspring of early-weaned d16 parents, compared to d26 offspring had greater d10-body-fat mass $(P<0.0001$ and 0.001 for females and males, respectively) and smaller lean body mass $(P<0.001$ and 0.05 , respectively; Figure $2 c)$.

\section{Developmental tempo}

Research on rodents shows that an animal's early rearing environment regulates pubertal, sexual and reproductive development [15]. To test the hypothesis that weaning age programs the tempo of development, F1 to F3 animals were observed from $\mathrm{d} 7$ to 20 for the following infantile developmental milestones [11]: fur budding (mean d9.2 milestone for control group d21), incisors eruption (d10.2), pinnae detachment (d12.1) and eye opening (d15.9). Whereas F1 animals showed no difference across the original age-of-weaning groups (data not shown), F2 offspring of early-weaned parents accelerated their pre-weaning infantile development as compared to offspring of late-weaned parents, showing earlier fur budding, pinnae detachment and eye opening (all $P<$ $.05)$, but not incisor eruption; Figure $3 \mathrm{a}, \mathrm{b}$ ). This difference in developmental tempo was sustained for F3 to F4 pups.

\section{Reproductive strategy}

The age at sexual maturation plays a central role in reproductive strategy. We thus predicted and found that early-weaned F2 females showed earlier vaginal opening $(P<0.01)$ and estrous $(P<0.01)$ and early-weaned males showed earlier onset of testicular growth $(P<0.05)$ and attainment of maximal testicular volume $(P<0.05)$ compared to late-weaned rats (Figure 3c, d). Moreover, adult d16 animals had larger testes compared to the other two groups $(P<0.001)$. These traits were enhanced from F2 to F4 generations in both female (Figure 3c) and male rats (data not shown).

To further test for effects on reproductive-strategyrelated outcomes, we studied parity numbers and $\mathrm{d} 1$ litter size of early- and late-weaned parents (F1) and grandparents (F2), predicting that early-weaned progeny would themselves bear more offspring than the progeny of laterweaned F1 rats. In the primiparous F3 and F4 rats, parity number of early-weaned was greater than late-weaned animals $(P<0.05$ and $<0.01$, respectively) (Figure $4 \mathrm{a}, \mathrm{b})$. The d1 litter size was greater in early-weaned than lateweaned in F3 $(P<0.01)$ and F4 $(P<0.01)$. Finally, we discovered that $\mathrm{d} 16$ were heavier than $\mathrm{d} 26$ pups in F3, $P<$ 0.02 , and in F4 litter, $P<0.04$ upon examining the mean weight of pups for each group after dividing $\mathrm{d} 1$ litter size by parity number. 

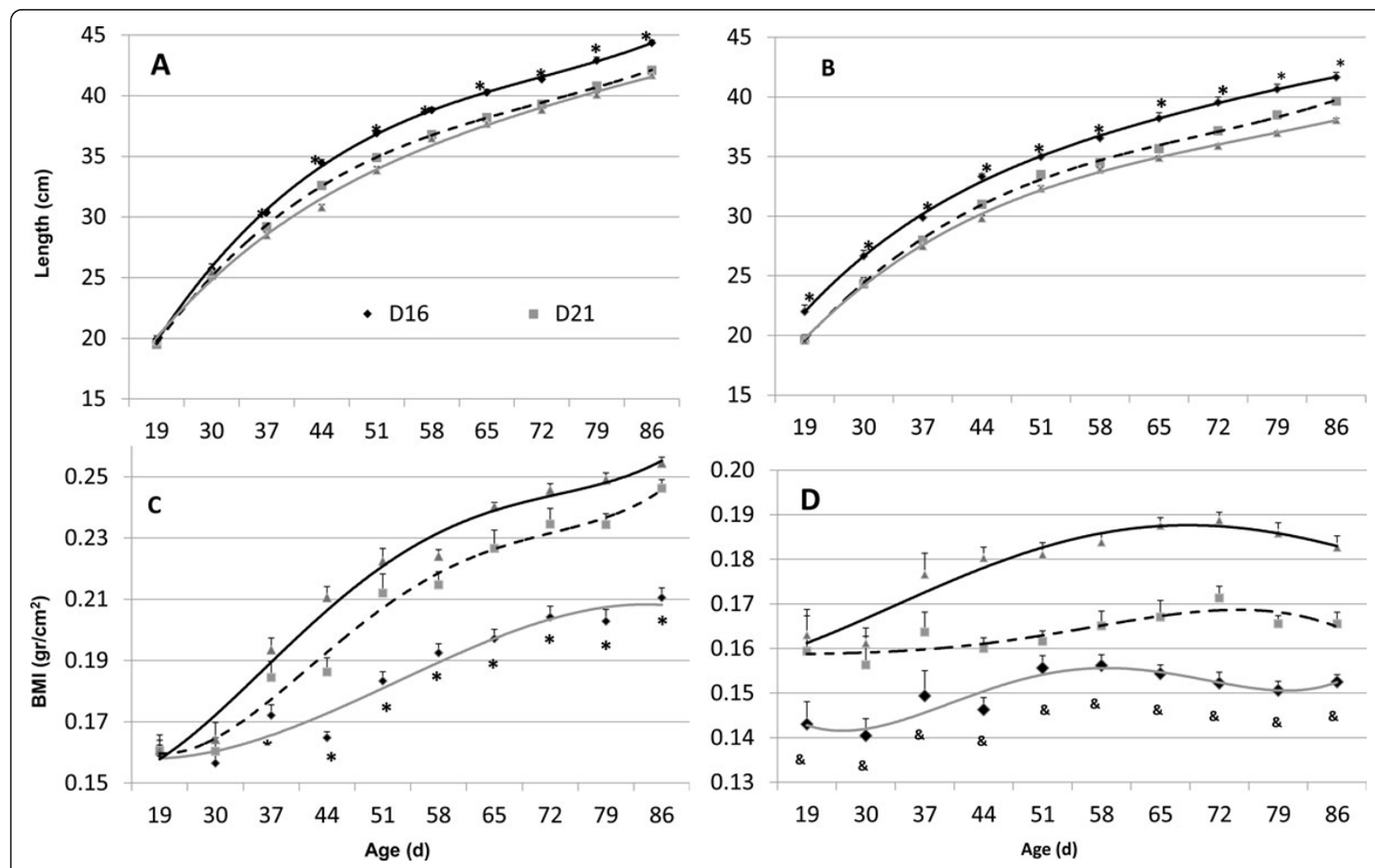

Figure 1 Growth phenotype according to weaning age. Length and body mass index (BMI) of rats of F1 generation according to weaning age day (d) 16, 21 or 26: Males (A, C) and females (B, D) were measured for crown-tale length (A, B) and weight to give the BMl (g/cm2; C, D) from age d19 to 90 . Statistically significant differences between $\mathrm{d} 16$ and d26 rats are indicated by $(\&)$ for $P<0.02$, $\left(^{*}\right)$ for $P<0.001$ with a twoway t-test. Mean $\pm \mathrm{SEM}, n=12$ per group.

\section{Phenotypic discordance upon paternal or maternal transmission}

When we evaluated the relative influence of mothers' and fathers' own age of weaning (as pups) on the weaningrelated phenotype of their offspring - after mating (a) F1 females that had weaned on d21 with F1 males that had weaned on d16 or d26 and (b) F1 males that had weaned on d21 with F1 females that had weaned on d16 or d26only paternal influence proved evident (Figure 5): growth was faster $(P<0.01)$ and BMI was smaller $(P<0.001)$ in F2 offspring of F1 fathers weaned on d16 as compared to fathers weaned on d26 (Figure 5A-D); and testicular growth was greater $(P<0.001)$ and vaginal opening $(P<$ $0.01)$ and estrous were earlier $(P<0.01)$ in F2 offspring of F1 fathers, weaned on d16 as compared to fathers weaned on d26 (Figure 5E-G).

\section{Discussion}

The findings presented here on rats lend support to the proposition that the duration of infancy, as indexed by weaning age, predicts and perhaps programs growth, body composition, and the tempo of physiological development and maturation, as well as litter size and parity, and, thereby, reproductive strategy.

Two important environmental cues for development of the young animal (and humans) are 1) the care-giving behaviors of their parents, which can be used as a predictive indicator of the security of their environment, and 2) provision of nutrition during the immediate postnatal period, which may predict nutritional availability during future life. The resultant patterns will be transmitted trans-generationally $[16,17]$. In view of prior evidence that weaning in rats has unique and critical effects on adult behavior [18], the data presented here indicate that weaning age influenced development in a manner consistent with an insecure, fast, life-history strategy (15), including accelerated growth, development and maturation, long/thin stature and, in subsequent generations, large litter size. In contrast, prolonged lactation influenced development in a manner consistent with a secure, slow, life-history strategy, including slower growth, development and maturation, short/overweight stature, and, in subsequent generations, small litter size. Yet, under natural conditions, early weaning might well be associated 

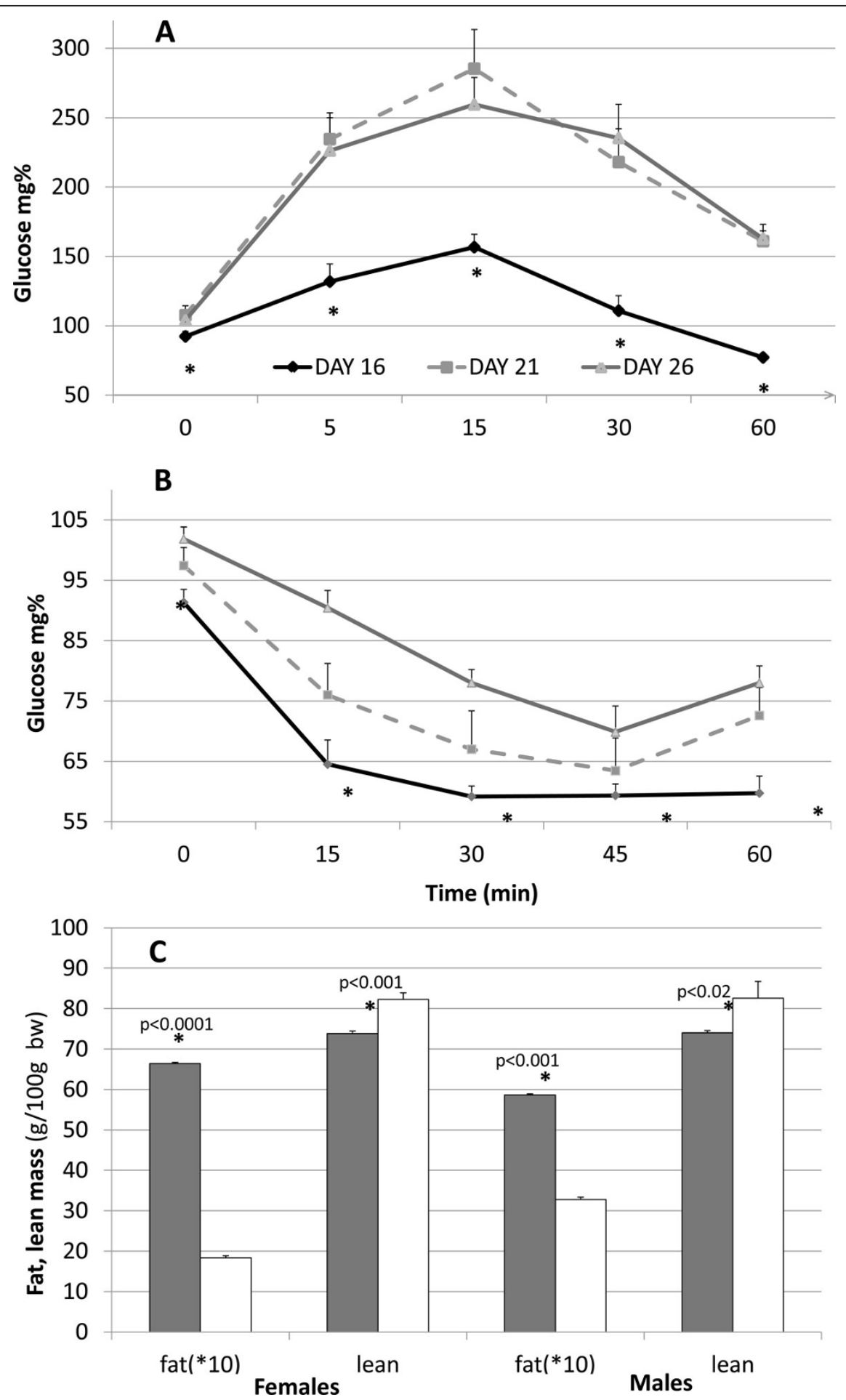

Figure 2 Glucose tolerance, insulin sensitivity and pups body composition. Glucose tolerance, insulin sensitivity and pups body composition according to weaning age d16, d21 or d26 in male adult F1 rats on d80 to 90. (A). Enteral glucose tolerance test: after six hours fasting, $0.1 \mathrm{~g} / \mathrm{ml}$ glucose was given intragastic through a feeding tube to provide $1 \mathrm{~g}$ glucose for each $100 \mathrm{~g}$ body weight. (B) Insulin tolerance test: Rats were injected intraperitoneally with insulin $(0.75 \mathrm{U} / \mathrm{kg})$ and blood glucose was measured by glucometer. (C) For assessment of body composition, d10 rats of F2 generation, whose parents weaned on d16 (dark bars) or d26 (white bars), were tested by live-mice TD-NMR Analyzer. The onboard electronics calculated fat and lean (mostly muscle) mass per $100 \mathrm{~g}$ body weight (\% fat is multiplied by 10 for clarity). Statistically significant differences between $\mathrm{d} 16$ and $\mathrm{d} 26$ rats are indicated by ${ }^{*}$ ) for $P<0.05$ with a two-way t-test. Mean \pm SEM, $n=6$ per group. 


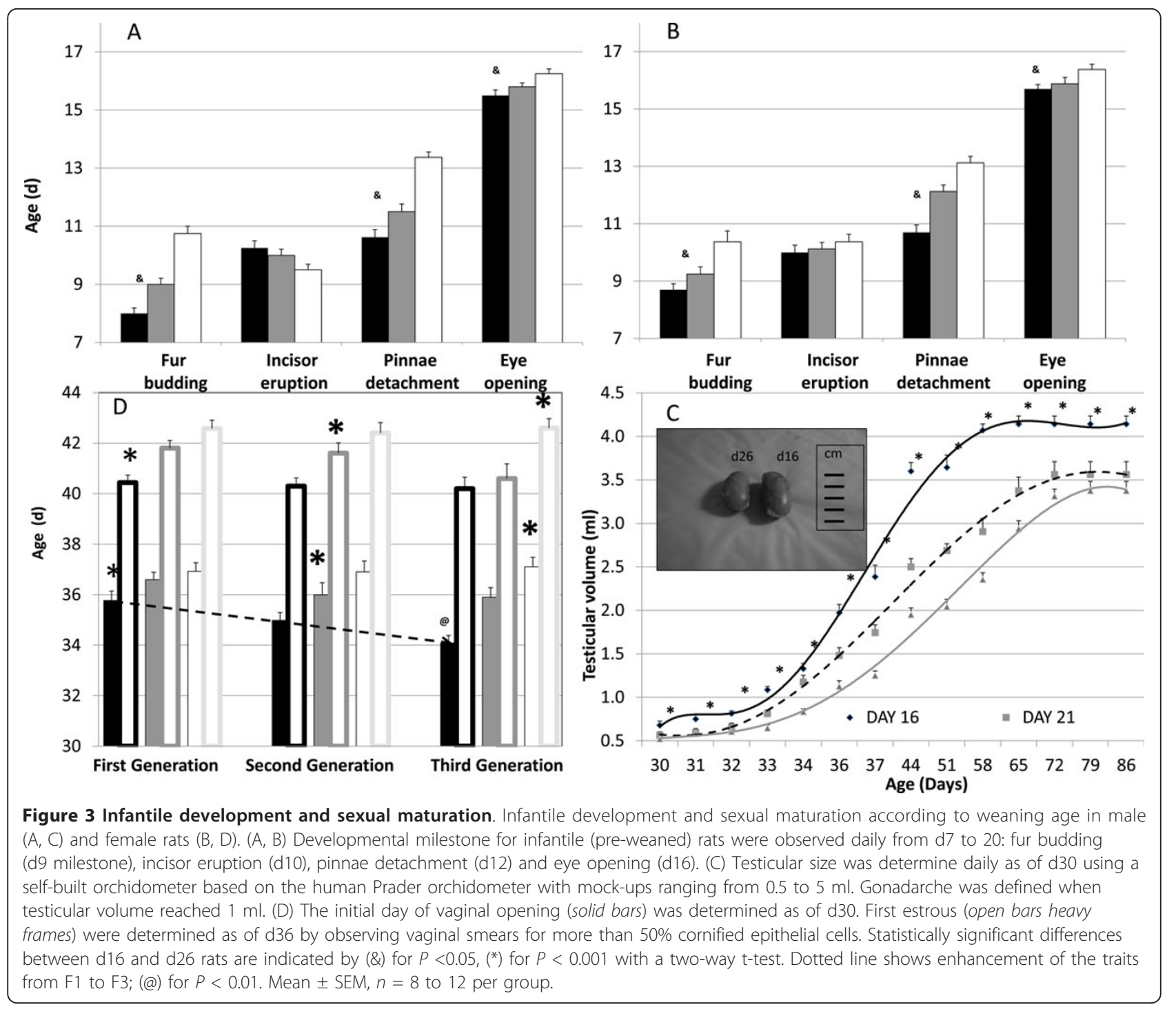

with more robust nutritional conditions that lead to more rapid pup growth and an earlier attainment of an appropriate size for independence. Similarly, in humans living in subsistence ecological contexts, early weaning is associated with better nutritional conditions.

The growth-promoting effect in rats of short infancy is much in line with human observations on the growth impact of early ICT $[4,5]$. We have proposed that adult size is determined to an important extent during transition from infancy to childhood. This transition is marked by a growth spurt. A delayed transition has a lifelong impact on stature and is responsible for $44 \%$ of children with short stature in developed countries, and many more in developing countries. This conformed with the theory of an evolutionary adaptive strategy of plasticity in the timing of transition from infancy into childhood in order to match the prevailing energy supply: humans evolved to withstand energy crises by decreasing their body size, and evolutionary short-term adaptations to energy crises trigger a predictive adaptive response that modify the transition into childhood, culminating in short stature $[1,6]$.

The apparent tempo-accelerating effect in infantile developmental milestones of parental short lactation in the rat indicates a developmental signal transmitted across generations and seemingly aimed to prepare the young animal for independence for provision and protection upon weaning. This raises the interesting question of exactly how short lactation accelerates development, as we have provided evidence that it does, not of the underlying neurobiological mechanisms involved. Future work will need to illuminate such processes. In any event, it is of interest that early sexual maturation and large litters in animals are in line with an accelerated life history tempo brought about by shortening of the infancy stage. In swine, sows' parity number and litter size increase as 


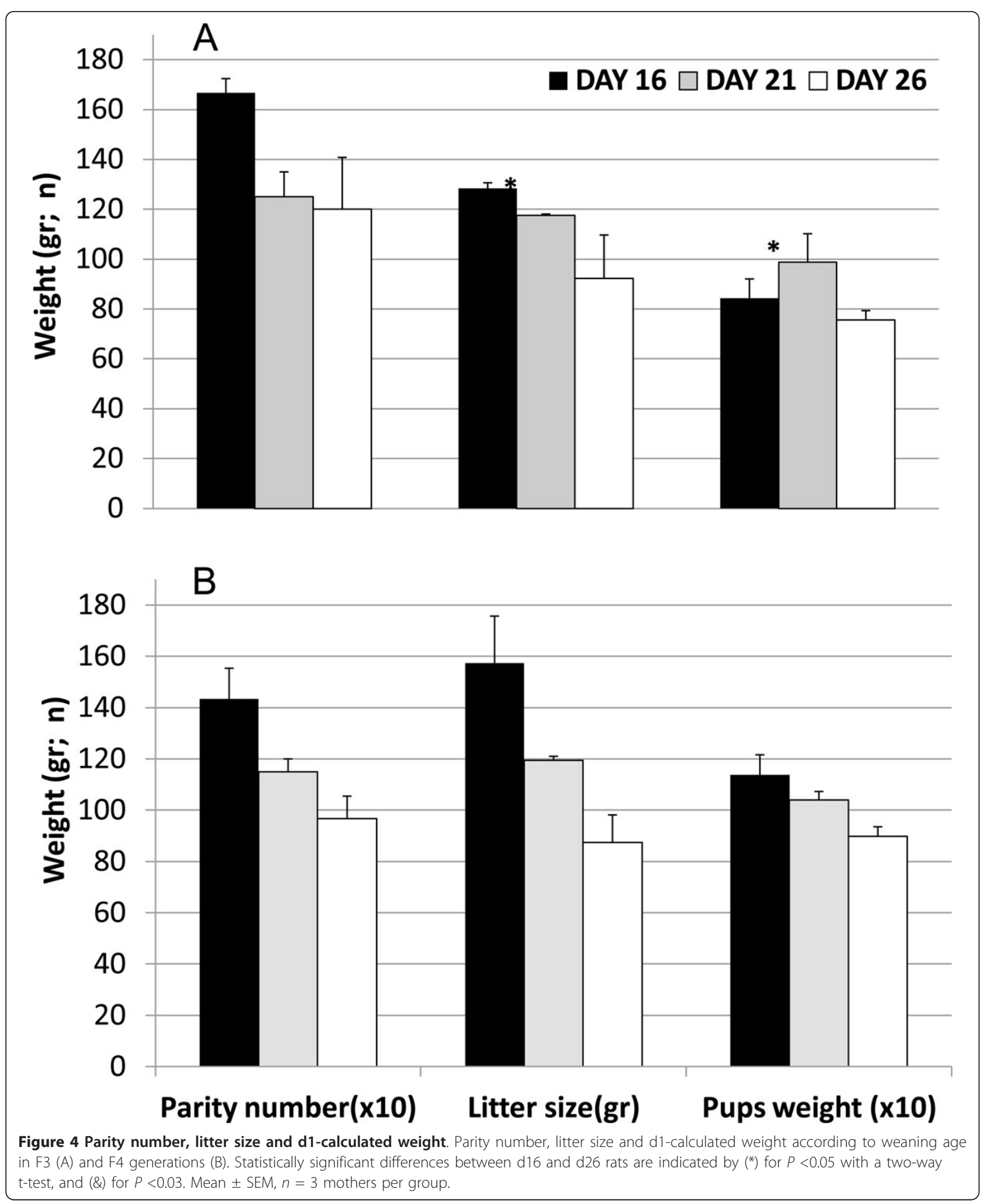

lactation is prolonged from 8 to 13 to 18 to 21 days, but decrease if lactation is further prolonged to 22 to 25 days [19], in agreement with the rat findings.
Asymmetries in the costs and benefits of parental investment for mothers and fathers result in family conflict over their offspring's growth [20]. In species where 


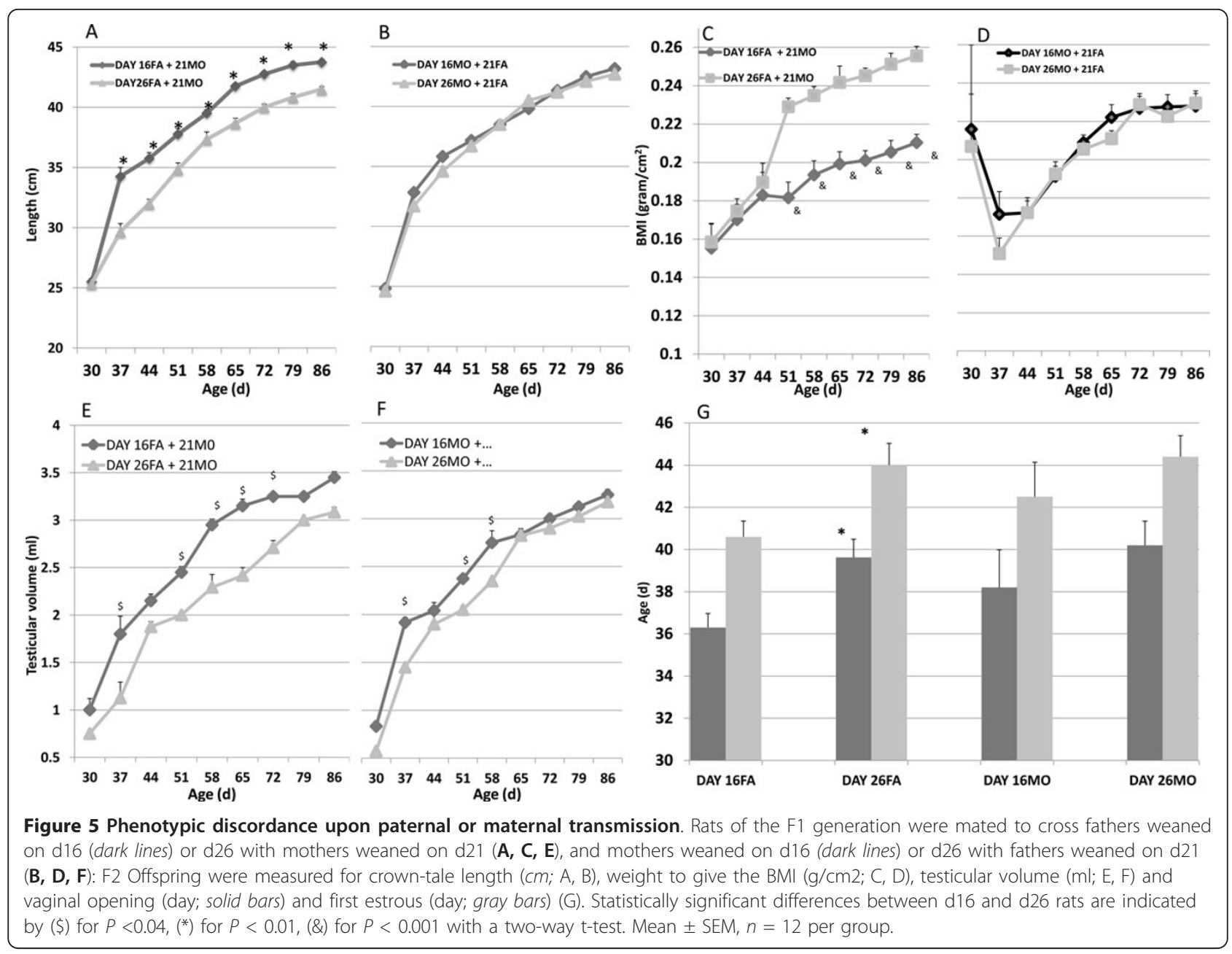

females provide most resources before and after birth, the resolution of this conflict may be influenced by genes expressed in mothers and by maternally and paternally inherited genes expressed in offspring [21]. Here we show that the weaning-related trait is transmitted from the paternal side; offspring of fathers but not of mothers who weaned early were longer, thinner and had earlier sexual maturation. Previous work in mice showed that differences in litter size are determined by paternal genotype, whereas differences in provisioning are under maternal control, suggesting that there is antagonistic coadaptation of maternal and paternal effects on distinct life-history traits [21]. These results are consistent with a negative correlation that we discerned between the age of the infancy-childhood growth transition and fathers' height but not mothers' heights [6], leading us to conclude that the trans-generational transmission of transition age appears paternally derived.

The fact that the glucose intolerance and insulin resistance were related to delayed weaning is considered a response to being overweight. The thrifty phenotype theory suggests that intrauterine wasting and early infantile growth acceleration are associated with later acquisition of obesity and insulin resistance [22]. Indeed, on d10 offspring of late weaned animals, which are to become overweight as adults, had diminished adipose tissue, suggesting that the adult phenotype may be influenced by infantile expansion of the adipose tissue [23].

Considered together, the animal results reported support a conditional-adaptational view of individual differences in the infantile stage: developmental tempo and pubertal maturation are accelerated adaptively in response to shortening of infancy to allow for juvenile independence upon early weaning (development) and earlier reproduction (sexual maturation) [24].

\section{Conclusions}

In the discussion of nature and nurture, our results lend support to a major impact of a conditional adaptation within a single generation, and provide insight into the role of plasticity over one-to-three generations in reproductively important traits such as size, developmental and 
maturational tempo. The notion that developmental tempo is regulated by weaning age to produce such contrasting phenotypes, and that fathers transmit the trait, has significant implications for evolutionary biology as well as to child growth and maturation in a changing society.

\section{Abbreviations}

BMI: Body mass index; ICT: the infancy-to-childhood transition.

\section{Authors' contributions}

YC and ZH designed and YC, OK, DBY and ZH performed the study. $Y C$ and $\mathrm{ZH}$ contributed to writing of the manuscript. All authors have read and approved the manuscript for publication.

\section{Competing interests}

The authors declare that they have no competing interests.

\section{Author details}

'Division of Pediatric Endocrinology, Meyer Children's Hospital, Rambam Health Care Campus, Haaliya Street, Haifa 31096, Israel. ${ }^{2}$ Endocrine Laboratory, Rambam Health Care Campus, Haaliya Street, Haifa 31096, Israel. ${ }^{3}$ Rappaport Family Faculty of Medicine, Technion - Israel Institute of Technology, Efron Street, Haifa 31096, Israel.

Received: 5 September 2012 Accepted: 20 December 2012 Published: 29 April 2013

\section{References}

1. Hochberg Z, Feil R, Constancia M, Fraga M, Junien C, Carel JC, Boileau P, Le Bouc Y, Deal CL, Lillycrop K, Scharfmann R, Sheppard A, Skinner M, Szyf M. Waterland RA, Waxman DJ, Whitelaw E, Ong K, Albertsson-Wikland K: Child health, developmental plasticity, and epigenetic programming. Endocr Rev 2011, 32:159-224.

2. Bogin B: Evolutionary perspective on human growth. Annu Rev Anthropol 1999, 28:109-153.

3. Smith B: Life history and the evolution of human maturation. Evol Anthropol 1992, 1:134-142.

4. Hochberg Z: Evo-devo of child growth II: human life history and transition between its phases. Eur J Endocrinol 2009, 160:135-141.

5. Hochberg Z, Albertsson-Wikland K: Evo-devo of infantile and childhood growth. Pediatr Res 2008, 64:2-7.

6. Hochberg Z: Evo Devo of Child Growth: Treatize on Child Growth and Human Evolution New York, NY: Wiley; 2012.

7. Cook CJ: Patterns of weaning and adult response to stress. Physiol Behav 1999, 67:803-808.

8. Caldji C, Diorio J, Meaney MJ: Variations in maternal care alter GABA(A) receptor subunit expression in brain regions associated with fear. Neuropsychopharmacology 2003, 28:1950-1959.

9. McGowan PO, Suderman M, Sasaki A, Huang TC, Hallett M, Meaney MJ, Szyf M: Broad epigenetic signature of maternal care in the brain of adult rats. PLoS One 2011, 6:e14739.

10. Denenberg VH, Grota LJ, Zarrow MX: Maternal behaviour in the rat: analysis of cross-fostering. J Reprod Fertil 1963, 5:133-141.

11. Heyser CJ: UNIT 8.18 Assessment of developmental milestones in rodents. Current Protocols in Neuroscience Hoboken, NJ: Wiley-Blackwell; 2003, 8.18.11-18.18.15.

12. Prader A: Testicular size: assessment and clinical importance. Triangle 1966, 7:240-243.

13. Halldorsdottir S, Carmody J, Boozer CN, Leduc CA, Leibel RL: Reproducibility and accuracy of body composition assessments in mice by dual energy $x$-ray absorptiometry and time domain nuclear magnetic resonance. Int J Body Compos Res 2009, 7:147-154.

14. Bruning JC, Winnay J, Bonner-Weir S, Taylor SI, Accili D, Kahn CR: Development of a novel polygenic model of NIDDM in mice heterozygous for IR and IRS-1 null alleles. Cell 1997, 88:561-572.

15. Cameron NM, Champagne FA, Parent C, Fish EW, Ozaki-Kuroda K, Meaney MJ: The programming of individual differences in defensive responses and reproductive strategies in the rat through variations in maternal care. Neurosci Biobehav Rev 2005, 29:843-865.

16. Belsky J, Fearon RM: Early attachment security, subsequent maternal sensitivity, and later child development: does continuity in development depend upon continuity of caregiving? Attach Hum Dev 2002, 43:361-387.

17. Del Giudice M: Sex, attachment, and the development of reproductive strategies. Behav Brain Sci 2009, 32:1-21, discussion 21-67.

18. Zimmerberg $B$, Weston HE: Postnatal stress of early weaning exacerbates behavioral outcome in prenatal alcohol-exposed juvenile rats. Pharmacol Biochem Behav 2002, 73:45-52.

19. Costa E, Amaral Filha WS, Costa AHA, Carvalho FF, Santos AK, Silva AF: Influence of the lactation length in the subsequent litter size of sows. Anim Reprod 2004, 1:111-114.

20. Haig D: Colloquium papers: Transfers and transitions: parent-offspring conflict, genomic imprinting, and the evolution of human life history. Proc Natl Acad Sci USA 2010, 107(Suppl 1):1731-1735.

21. Hager $R$, Johnstone RA: The genetic basis of family conflict resolution in mice. Nature 2003, 421:533-535.

22. Neel JV: Diabetes mellitus: a "thrifty" genotype rendered detrimental by "progress"? Am J Hum Genet 1962, 14:353-362.

23. Wells JC: The thrifty phenotype hypothesis: thrifty offspring or thrifty mother? J Theor Biol 2003, 221:143-161.

24. Belsky J, Steinberg L, Draper P: Childhood experience, interpersonal development, and reproductive strategy: and evolutionary theory of socialization. Child Dev 1991, 62:647-670.

doi:10.1186/1741-7015-11-114

Cite this article as: Crispel et al:: Effects of breastfeeding on body composition and maturational tempo in the rat. BMC Medicine 2013 11:114.

\section{Submit your next manuscript to BioMed Central and take full advantage of:}

- Convenient online submission

- Thorough peer review

- No space constraints or color figure charges

- Immediate publication on acceptance

- Inclusion in PubMed, CAS, Scopus and Google Scholar

- Research which is freely available for redistribution
C Biomed Central 